\title{
Research on Effects of Herb of Glabrous Sarcandra on Human Prostate Carcinoma DU-145 Cells PI3K I m TOR Signal Transduction Pathway
}

\author{
Xiuxia Wang \\ Luohe Medical College, Luohe, Henan, China, 462000
}

\begin{abstract}
Keywords: Herb of Glabrous Sarcandra, Human Prostate Carcinoma, DU-145 Cells, PI3K / m TOR Signal Transduction Pathway
\end{abstract}

\begin{abstract}
In order to investigate the effect of herb of glabrous sarcandra solution on human prostate cancer DU-145 cells PI3K / m TOR signal transduction pathway, the paper uses method of cell culture, with Sarcandrae solution with different concentrations of 48h, the impact on DU-145 cells were detected by MTT solution herb of glabrous sarcandra method of DU-145 cell proliferation by RT - PCR detected after treatment cells PI3K, mTOR and P70S6K gene expression. Herb of glabrous sarcandra action of wind solution after 48h, DU-145 cell proliferation was inhibited and showed dose-effect relationship; DU-145 cells expressing the P13K, mTOR and P7OS6K showed different degrees of inhibition, it does not show a dose relationship. This indicates herb of glabrous sarcandra solution can inhibit human prostate cancer DU-145 cell proliferation, cell proliferation inhibition mechanism related to the inhibition of cell P13K / mTOR signaling pathway effects.
\end{abstract}

\section{Introduction}

Prostate Cancer Prostate cancer is the most common variety - species, is currently the world's four most common cancers in men, and the incidence is rising. The incidence of prostate cancer in the world there is a great difference, the main difference is that the incidence of clinical morbidity, with the highest incidence in Europe and America countries, accounting for the first two male cancer in the United States the incidence of prostate cancer in men accounts malignant first one. The incidence of prostate cancer is although much lower than the United States and Europe, but showed a significant growth trend. Provided in 1999 in Paris, held the second session of the International Advisory Committee of prostate cancer the 1988--1992 years, the incidence of prostate cancer in the International, where the highest incidence of black Americans was 137/10 million men, while the lowest is our Shanghai 2 / 10 million men, a difference of 60 times. However, prostate cancer is 1973--2005 years, the Shanghai area common male cancer standardized incidence rate (age-adjusted standardized) rising the fastest, reaching 5.96\%, especially in the 20 years 1986-2005, standardized incidence rate increased to reach $10.04 \%$. Elderly men with prostate cancer are the most common malignant tumor and more than $95 \%$ of cases are diagnosed at the age of 45 to 89 years and mean age was 72 years. In addition to clinical onset of prostate, there are at necropsy or do other prostate examination revealed prostate cancer called latent cancer, men over the age of 70 up to 25 percent of latent cancer, the development of clinical cancer accounts for only a very small section. Fully visible high incidence of prostate cancer, and showed an increasing trend.

\section{Traditional Chinese Medicine Pharmacological Study of Herb of Glabrous Sarcandra}

Anti-Tumor Effect. Through injectable liquid outer anti-tumor effect, the results show herb of glabrous sarcandra injection on human lung cancer cell A-149, HCT-29 colon cancer cells and gastric cancer cells BGC-823 three human tumor cells are inhibited. Experimental study of herb of glabrous sarcandra sheet showed anti-tumor effect, herb of glabrous sarcandra sarcoma S180 inhibition rate of $27.00 \%-29.00 \%$; solid tumors of HepA inhibition rate was $25.00 \%-36.25 \%$. In vivo, in vitro two methods herb of glabrous sarcandra injection on mouse hepatoma HepA-22 and its effects on body weight of nude mice showed that sarcandrae vitro and in vivo in mice liver cancer HepA-22 anti-tumor effect. Another experiment showed that the former have a mouse gastric anti-tumor effect in vivo. Studies have shown that herb of glabrous sarcandra with 
chemotherapy (CTX, 5-FU, AOM) and radiotherapy (60Co irradiation) and treated with anti-tumor effect can be increased and efficiency was $11.17 \%$-39.80\%. It adriamycin on HCT-8 cells can produce additive or synergistic inhibitory effect enhanced, especially herb of glabrous sarcandra injection with high concentrations of doxorubicin synergistic effect is more pronounced.

Anti-Viral, Anti-Bacterial and Anti-Inflammatory and Analgesic Effects. Vivo tests showed Herb of glabrous sarcandra influenza virus A, Beijing Branch 168 are inhibited to cause respiratory infections kill most bacteria and significantly inhibited. The results showed that Herb of glabrous sarcandra croton oil-induced mouse ear inflammation Guo, carrageenan-induced rat paw hesitate inflammation and granuloma in mice have significant inhibition. In addition, it can significantly reduce the pain caused by acetic acid and inhibit bacterial growth. Description Herb of glabrous sarcandra has significant anti-bacterial, anti-inflammatory and analgesic effects. Huang Fang and different concentrations herb of glabrous sarcandra extract is added Streptococcus mutans medium at different concentrations of drug in the medium transferase activity inhibition diameter, bacterial cell number and $\mathrm{pH}$ changes and glucosyl, to examine herb of glabrous sarcandra extract on Streptococcus mutans in vitro cariogenic force, the results show sarcandrae inhibit bacterial growth, inhibit glucosyl transferase activity and the like.

Effect on White Blood Cells and Platelets. Herb of glabrous sarcandra $60 \%$ alcohol extract can very significantly shorten the mouse tail bleeding time and clotting time, strengthen the platelet contractile function, no significant effect on normal platelet count; for Ara $\mathrm{P}$ induced thrombocytopenia and leukopenia significantly therapeutic effect. The results showed that herb of glabrous sarcandra site and I have to upgrade the role of platelets, sarcandrae animals adrenal, thymus weight no significant changes in parts I thymus weight significantly. Description herb of glabrous sarcandra anti-immune thrombocytopenic purpura role sarcandrae separation site I was part of the effective anti-immune thrombocytopenic purpura.

\section{Culture and Preservation of DU-145 Human Prostate Cancer Cells}

Training and Change of Human Prostate Cancer Du-145 Cells. After the repurchase of DU-145 cells, the flasks original aspirating liquid, containing 10\% fetal calf serum RPMI-1640 medium, no more than $5 \mathrm{ml}$, with a surface of $75 \%$ ethanol graze flasks after placed 37e, 5\% CO2 the incubator culture medium was changed every 2-3d - times. Daily routine changes in cell morphology were observed under inverted microscope, and to observe the extent of cell fusion, cell fusion to be $80 \%$ for passage.

Subculture of Human Prostate Cancer DU-145 Cells. Cell fusion to be more than 80\% were passaged, the flasks were removed from the incubator, clean bench in the flasks with liquid disposable pipette, then add D-Hank'S balance was washed 2-3 times, rinse flask residual serum, $0.25 \%$ trypsin $2-3 \mathrm{ml}$, digestion at room temperature $3-4 \mathrm{~min}$, and continue to observe the cells under an inverted microscope, until most of the cells became round, floating, instantly disposable suction flasks trypsin added RPMI-1640 medium containing 10\% fetal bovine serum to terminate digestion, and continue pipetting cells, the cells were completely off the wall, made of cell suspension, and then the human prostate cancer DU-145 cells were placed in culture flasks was added 10\% fetal bovine serum containing RPMI-1640 medium, into 37e, 5\% COZ incubator incubation. Subculture interval is 2-3 times per week, passage ratio of 1: 3 .

The Frozen of Human Prostate Cancer Du-145 Cells. Good cell growth and high density, removing the growth solution, PBS rinsed three times. Added $0.25 \%$ trypsin for $2-5$ minutes, then add cryopreservation solution with pipetting, the cells were uniformly dispersed and counted using a cell freezing medium to the cell concentration was adjusted to $2 * 106 / \mathrm{m} 1$, load cells frozen custody, with about $2 \mathrm{~cm}$ thick gauze wrapped vials, ultra-low temperature freezer for 24 hours and then transferred to -150.C refrigerator frozen.

\section{Cell Morphology of Du-145}

Preparation of Specimens. With $0.25 \%$ trypsin digestion flasks logarithmic phase of DU-145 cells, 
which moved within a 6-well plate (built-in cover glass by pickling, sterilization treatment) adherent growth. Let it crawl sheet growth of about 60\%, for LY294002 was added to a solution of various concentrations (2.smg / ml, 1.25mg / ml, $0.625 \mathrm{mg} / \mathrm{ml}$ ) and a solution of Herb of glabrous sarcandra of $25 \mathrm{pmol} / \mathrm{l}$. Respectively, for 48 hours, remove the coverslip, spare.

Morphology. With an ordinary optical microscope (40X), focusing on changes in cell morphology was observed, whether cell size smaller, nuclear condensation occurs whether or fragmentation.

\section{Experimental Results and Analysis}

The Change of Herb of Glabrous Sarcandra on DU-145 Cell Morphology. DU-145 human prostate cancer cells were herb of glabrous sarcandra solution of each concentration group and LY294002 group after 48h treatment, mainly as exhibited significantly reduced the number of cells, the cell gap increases significantly, cell sizes, irregular shape, the overall volume reduced. Fine varying degrees of cell shrinkage, rough edges, chromatin condensation, nuclear condensation or fragmentation. Among herb of glabrous sarcandra solution 2.smg / $\mathrm{ml}$ concentration group the most significant.

Herb of Glabrous Sarcandra of Du-145 in Cell Proliferation. OD higher the value, the more the number of viable cells, indicating that the stronger the proliferation inhibition worse.

The negative control group, OD value was significantly higher than the control group $(\mathrm{P}<0.01)$, DU-145 cells show active proliferation; Herb of glabrous sarcandra solution $2 . \mathrm{smg} / \mathrm{ml}, 1.25 \mathrm{mg} / \mathrm{ml}$ and LY29400225 forest mol / $\mathrm{ml}$ OD concentrations were lower than the negative control group, and showed a highly significant difference $(\mathrm{P}<0.01)$, Sarcandrae solution $0.625 \mathrm{mg} / \mathrm{ml}$ concentration group OD values are different than the negative control group $(\mathrm{P}<0.05)$. Low concentration group and LY29400225 forest mol / ml concentration group Herb of glabrous sarcandra solution 2.smg / ml inhibitory potency of the high dose group of DU-145 cells was significantly higher than Herb of glabrous sarcandra $1.25 \mathrm{mg} / \mathrm{ml}, 0.625 \mathrm{mg} / \mathrm{m} 1$ of ( $<<0.01$ ), while Sarcandra, the low-dose group and LY29400225 fight mol / ml concentrations of the inhibitory potency of DU-145 cells were substantially equivalent, no statistical difference. The OD value of the DMSO solution in the control group was significantly lower than the negative control group.

The results show that the solution Sarcandrae high, medium and low concentrations can inhibit the proliferation of DU-145 cells, its ability to inhibit cell showed a dose-effect relationship, high concentrations inhibit the ability of DU-145 cells was significantly stronger than low concentration group and LY29400225 forest mol / $\mathrm{ml}$ concentration group. The OD value of the DMSO solvent control group was lower than the negative control group reasons may be DMSO itself to cells given the toxicity.

\section{The Vitro of Herb of Glabrous Sarcandra on Human Prostate Cancer DU-145 Cells}

On DU-145 Cell Morphology. DU-145 human prostate cancer cells were Herb of glabrous sarcandra solution of each concentration group and LY294002 group 48h after treatment were mainly for the number of cells significantly reduced cell gap increases significantly, cell sizes, irregular, generally smaller in size; varying degrees of cell shrinkage, rough edges, chromatin condensation, nuclear condensation or fragmentation. Among herb of glabrous sarcandra solution $2.5 \mathrm{mg} / \mathrm{ml}$ high concentration group the most significant. Description herb of glabrous sarcandra wind suppression number of tumor cells, destroying the normal growth and promote their degeneration and death.

On DU-145 Cell Proliferation. Cell proliferation and apoptosis are normal growth of the organization, the exercise of the function of two basic elements. Tumor growth, invasion, metastasis and cell proliferation is closely related to its main dynamic characteristics of the hyperproliferative cells. Advances in cell proliferation and prostate cancer recurrence and prognosis of tumor cell proliferation is active directly affects the prognosis of tumors. 


\section{Effects of Herb of Glabrous Sarcandra on P13k / m TOR Signal Transduction Pathway.}

Based p13K / mTOR signaling pathway plays a crucial role in the occurrence and development of the HIPC, this research uses the currently used with fast, sensitive and accurate method and the three major advantages ELLISA RT.-PCR method, to discover herb of glabrous sarcandra the signal transduction pathway, which proved sarcandrae mechanism HIPC treatment.

By studying ELLISA method found herb of glabrous sarcandra solution with high, medium and low concentrations of the experimental group and LY294002 group concentration of human PI3K, mTOR and P70S6K were shown different degrees of inhibition, in which the three concentrations herb of glabrous sarcandra solution group inhibition of these substances does not reflect the dose-effect relationship. LY294002 positive drug test solution concentrations and inhibition potency herb of glabrous sarcandra the four substances were not statistically significant at three concentrations. It follows that the solution sarcandrae ear and 294,002 pairs of people PI3K, mTOR and P7oS6K inhibited substantially equivalent. The experiments show herb of glabrous sarcandra solution passed all inhibition PI3K, mTOR and P7oS6K4 substance concentration, thus affecting the PI3K / mTOR signaling pathway.

By RT-PCR method found herb of glabrous sarcandra solution with high expression of medium and low concentration group and LY294002 group experimental concentrations of PI3K, mTOR and P70S6K genes were shown different degrees of inhibition, which sarcandrae three solution concentrations of these substances do not reflect inhibition dose-effect relationship. LY294002 solution three experimental concentrations comparable to the concentrations of the inhibitory potency and herb of glabrous sarcandra these four substances, there is no difference. It follows that Sarcandrae solution and LY294002 on PI3K, mTOR gene expression and inhibit P7oS6K substantially equivalent. The experiments show herb of glabrous sarcandra solution passed all inhibition PI3K, mTOR and P70S6K genes expression, thus affecting the PI3K / mTOR signaling pathway.

Fully visible, ELLISA method and RT-PCR method is completely concluded - caused, therefore herb of glabrous sarcandra of PI3K, mTOR signaling pathway inhibition effect of realistic, credible, it should be Herb of glabrous sarcandra treatment HIPC - the item important principle.

\section{Conclusion}

Hormone-dependent prostate cancer prostate cancer treatment is difficult. Chinese medicine in the treatment of this disease reflects a certain advantage. This experiment has chosen detoxification, chills path effect with anti-tumor, anti-infection and enhances immunity pharmacological action herb of glabrous sarcandra treatment of human androgen-independent prostate cancer DU-145 cell lines for 48 hours. In summary, sarcandrae solution by inhibiting PI3K, mTOR signaling pathway, thereby inhibiting the DU-145 human prostate cancer cell proliferation is an important mechanism HIPC its clinical treatment.

\section{Acknowledgements}

Fund Project: Study on the Effect of Herba Sarcandrae on Proliferation and Apoptosis of Prostate Cancer DU -145 Cell the National Social Science Fund Project (152300410015);

\section{References}

[1] Jia Xinzhang, Li Jingyuan. Herbal Medicine, Vol. 6 (2014) No 53, p.25-26

[2] Wang Yunhui, Wang Qunyong. Chinese Medicine, Vol. 12 (2015) No 27, p.74-76

[3] Jing Jianfen. Zhejiang Traditional Chinese Medicine, Vol. 30 (2014) No 19, p.144-145

[4] Wang Kuailiang. Journal of Physiological Sciences, Vol. 29 (2010) No 27, p.21-23

[5] Zhang Gongxu, Sun Jing. Shanxi Journal of Medicine, Vol. 8 (2013) No 27, p.57-60 